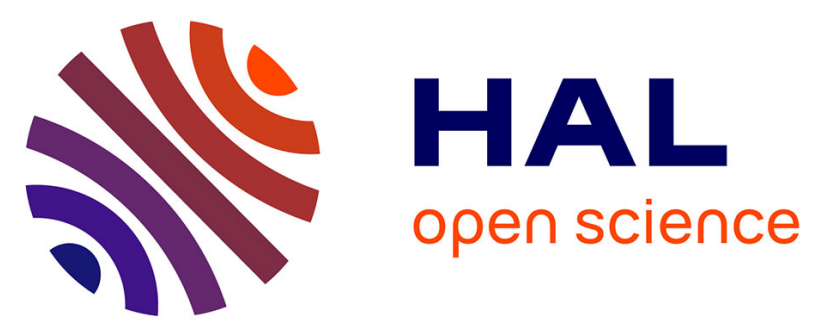

\title{
In-situ characterisation of the dynamics of a growing dust particle cloud in a direct-current argon glow discharge
}

S Barbosa, L Couëdel, C Arnas, Kalathiparambil Kishor Kumar, C Pardanaud, F R A Onofri

\section{To cite this version:}

S Barbosa, L Couëdel, C Arnas, Kalathiparambil Kishor Kumar, C Pardanaud, et al.. In-situ characterisation of the dynamics of a growing dust particle cloud in a direct-current argon glow discharge. Journal of Physics D: Applied Physics, 2015, 49 (4), pp.045203. 10.1088/0022-3727/49/4/045203 . hal-01451227v2

\section{HAL Id: hal-01451227 \\ https://hal.science/hal-01451227v2}

Submitted on 7 Jan 2022

HAL is a multi-disciplinary open access archive for the deposit and dissemination of scientific research documents, whether they are published or not. The documents may come from teaching and research institutions in France or abroad, or from public or private research centers.
L'archive ouverte pluridisciplinaire $\mathbf{H A L}$, est destinée au dépôt et à la diffusion de documents scientifiques de niveau recherche, publiés ou non, émanant des établissements d'enseignement et de recherche français ou étrangers, des laboratoires publics ou privés. 


\title{
In-situ characterisation of the dynamics of a growing dust particle cloud in a direct-current argon glow discharge
}

\author{
S. Barbosa ${ }^{1}$, L. Couëdel ${ }^{2}$, C. Arnas ${ }^{2}$, Kishor Kumar K. ${ }^{2} \ddagger$, \\ C. Pardanaud ${ }^{2}$, F.R.A. Onofri ${ }^{1}$ \\ ${ }^{1}$ Aix-Marseille Université, CNRS, IUSTI, UMR 7343, 13453 Marseille cedex 13, \\ France. \\ ${ }^{2}$ CNRS, Aix-Marseille Université, Laboratoire de Physique des Interactions \\ Ioniques et Moléculaires UMR 7345, 13397 Marseille cedex 20, France. \\ E-mail: lenaic.couedel@univ-amu.fr
}

\begin{abstract}
.
The growth and the dynamics of a tungsten nanoparticle cloud were investigated in a direct-current low pressure argon glow discharge. Real-time analyses of the dust particle size and number concentration were performed insitu by light extinction spectrometry, while spatial dynamics of the cloud was investigated with the laser light-sheet scattering method. Additional off-line electron microscopy and Raman spectroscopy measurements were also performed for comparison purpose. This experimental work reveals the existence of an agglomeration phase followed by the appearance of a new dust particle generation. While growing, the dust cloud is pushed towards the anode and the discharge edge. Afterwards, a new dust particle generation can grow in the space freed by the first generation of nanoparticles. The continuous growth, below the light extinction spectrometry scanning positions, explains the apparent dissimilarities observed between the in-line optical and the off-line electron microscopy analyses.
\end{abstract}

PACS numbers: 52.27.Lw, 52.25.Os, 52.40.Hf

Submitted to: J. Phys. D: Appl. Phys.

$\ddagger$ Present address: Center for Plasma-Materials Interactions, 216 Talbot Laboratory, 104 S Wright Street, University of Illinois at Urbana-Champaign, 61801 Illinois, USA 


\section{Introduction}

Dusty or complex plasmas are partially ionised gases which contain solid dust particles that acquire an electric charge due to their interactions with the surrounding ions and electrons. They can be either injected or grown directly inside the plasma. For example, nanoparticles (NPs) are produced as candidate analogue of dust in diffuse interstellar medium, in radio-frequency (RF) discharges using hydrocarbon gases [1]. In the context of dense plasmas, NPs were surprisingly found in dust samples collected in carbon-based wall tokamaks $[2,3]$. The possibility of formation in the coldest regions, near the tokamak wall was therefore investigated $[4,5,6]$.

Generally, NP formation [7, 8, 9, 10, 11], charging and transport $[12,13,14,15]$ were extensively studied experimentally in silane-based RF discharges for semi-conductor applications such as the improvement of photovoltaic devices [16]. It was shown that their growth started by the formation of molecular precursors which were coming from trapped negative ions resulting from the dissociation of reactive gases in the plasma $[17,18,19]$. After nucleation, NP growth was attributed to successive agglomerations of the smallest NPs. This mechanism was found to inhibit further nucleations resulting in NPs with narrow size dispersions [20,21]. Finally, the agglomeration stopped due to electrostatic repulsion and the growth continued at constant NP number concentration by surface deposition of molecular species.

It was recently demonstrated that in direct-current (DC) sputtering discharges, in which the nucleation was initiated by sputtered metallic atoms, the growth mechanisms were more complex [22]: i) small particles were always observed, indicating a constant nucleation and ii) the growth by agglomeration did not seem to saturate. Moreover, a strong dispersion in the appearance time of the second NP generation was reported [23]. These experiments were performed using a tungsten cathode as source of metal atoms. The incident DC discharge power being rather low, the cathode surface oxide was sputtered away after few tens of seconds. During this time interval, the evolution of the discharge and plasma parameters was the result of a competition between the influence of the cathode surface state and the influence of the charging of growing NPs. The influence of the latter became dominant on the plasma and discharge parameters (loss of plasma electrons) only when the oxide layer was removed [22]. Scattering of a vertical laser sheet going through the plasma just above the anode revealed that the NP cloud was compressed and pushed towards the anode during the discharge. Scanning electron microscopy (SEM) images of substrates exposed to the plasma for given durations showed that NPs were continuously falling down on the anode during the discharge [24]. These observations were explained by the fact that the electrostatic force at the negative glow-anode sheath boundary could not balance the ion drag, gravity, and thermophoresis forces for NPs of more than a few tens of nanometres in diameter. From electron microscopy images, it was shown that up to $\sim 500 \mathrm{~s}$, only mono-modal or bi-modal populations of NPs were collected [22].

However, in-situ characterization of the growing NP cloud at different heights between the cathode and the anode remained necessary to unveil completely the growth and transport processes of metallic NPs in a DC discharge. For this purpose, we decided to combine imaging of the NP cloud by the scattering of a laserlight sheet with light extinction spectrometry (LES) which permitted the direct measurement of the particle size distribution (PSD) and number concentration, $N$, of the NP cloud at different positions in the discharge. This diagnostic is based on the extinction of a collimated white light beam whose spectral range extended from ultra-violet to infra-red ranges. It has the capability to detect NPs smaller than $20 \mathrm{~nm}$ and is effectively used to measure PSDs and $N$ in colloidal suspensions [25]. This optical diagnostic presents various advantages with respect to the Mie-scattering ellipsometry diagnostic, often used in dusty plasmas $[15,21,26,27]$. In particular, in the latter case, it is experimentally more challenging as it requires accurate measurements of the ellipsometric angles and the iterative data procedure used to find the particle parameters (PSD, local density, refractive index) is not trivial and requires strong assumptions. Otherwise, a reduction of the number of parameters can be considered. For instance, the choice of a model providing the time evolution of the particle radius can help in this way [15, 21].

In the present paper, we report a complete study on the growth and transport of tungsten NPs in DC sputtering discharges, in similar conditions to our previous experiments $[22,23,24]$. Sections 2 and 3 are devoted to the description of the experimental 
set-up and different diagnostics (electron microscopy, Raman spectroscopy, laser-light scattering and LES). A special attention is given to the LES diagnostics whose resolution was numerically evaluated. Section 4 presents our experimental findings and finally, section 5 is an overall discussion with conclusions.

\section{Plasma-discharge: experimental set-up}

Tungsten NPs were grown in a DC argon glow discharge initiated between a $\sim 10 \mathrm{~cm}$ diameter tungsten cathode and a stainless-steal grounded anode (see Fig.1). The inter-electrode distance was also $10 \mathrm{~cm}$. Two glass half-cylinders were used to confine the plasma. A $1 \mathrm{~cm}$ gap was kept between them for optical diagnostics. A static argon pressure of 0.6 mbar (no gas flow) was set during the experiments. The electrode assembly was contained in a cylindrical vacuum chamber of $30 \mathrm{~cm}$ diameter and $40 \mathrm{~cm}$ length. An oil diffusion pump achieved a base pressure of $\leq 10^{-6}$ mbar. A manually operated gate valve was used to isolate the chamber from the oil diffusion pump during experiments.

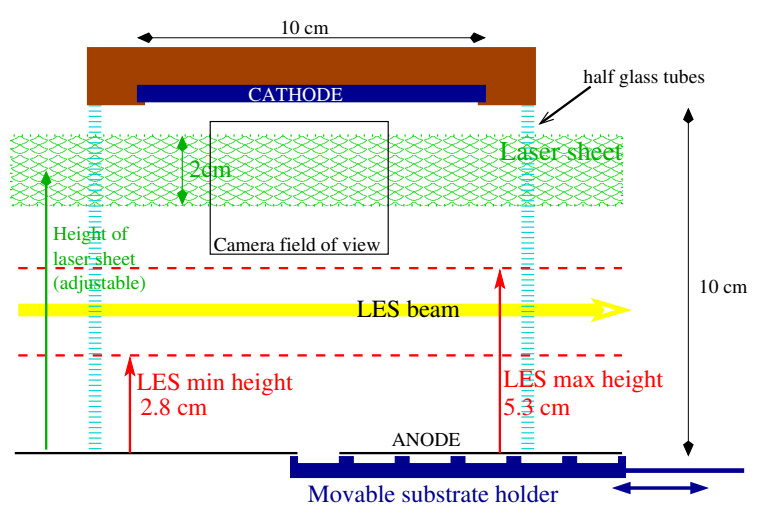

Figure 1. (Colour online) Schematic of the experimental set-up. The vertical position of the center of the laser-light sheet can be adjust between $1 \mathrm{~cm}$ and $9 \mathrm{~cm}$ above the anode. The field of view of the camera is adjusted to the laser-light sheet position. Note that the LES diagnostics and the laser-light scattering imaging were not implemented simultaneously.

A regulated power supply (Sefelec HT15A, $1 \mathrm{kV}$, $300 \mathrm{~mA}$ ) was used to bias the cathode. The discharge current density was kept at a constant value $\left(0.53 \mathrm{~mA} \cdot \mathrm{cm}^{-2}\right.$ corresponding to a current of $\left.40 \mathrm{~mA}\right)$. The current variations were less than $0.05 \%$. The discharge parameters were such that the plasma mainly consisted of a negative glow. Under the chosen operating conditions, the cathode was sputtered and tungsten NPs were grown. More details about the experimental set-up and the evolution of discharge parameters during NP growth are given in Ref.[22].

\section{Diagnostics}

\subsection{Electron microscopy}

The anode disc had a $1.6 \mathrm{~cm}$ diameter hole at the centre through which the tungsten NPs produced during the discharge were collected on stainless steel substrates. The substrate holder could be manipulated from one of the axial ports of the chamber, such that each substrate could be positioned under the hole in the anode. Two methods of collection were used:

- (i) The substrate holder was moved under the anode so that successive substrates were exposed to the plasma during a given time interval.

- (ii) The substrate saw the extinction of the discharge. Note that in this case, the substrate could, or not be exposed to the plasma during the whole discharge duration.

NPs were then analysed ex-situ using a scanning electron microscope. NPs have also been transferred from the stainless steal substrates to transmission electron microscopy (TEM) grid in order to perform high-angle annular dark-field imaging (HAADF) with a scanning transmission electron microscope (STEM). This technique, highly sensitive to atomic number variations in analysed samples, was used to check the tungsten content of the NPs.

\subsection{Raman spectroscopy}

Raman spectroscopy analyses were performed on collected NPs in order to determine if they were oxidised. The Raman spectrometer was a commercial Horiba-Jobin-Yvon HR LabRAM apparatus ( $\times 50$ lens, $\lambda_{R}=514.5 \mathrm{~nm}$, laser power at the surface $\left.\approx 1 \mathrm{~mW} \cdot \mu \mathrm{m}^{-2}\right)$. This technique consists in measuring the wavelengths of photons produced by inelastic scattering of the incident light with the analysed sample. The energy difference between incident and scattered photons corresponds to a vibrational energy which can be used as a fingerprint of a given material. Tungsten oxides are known to display optical Raman active bending and stretching modes in the ranges 100$300 \mathrm{~cm}^{-1}$ and $700-800 \mathrm{~cm}^{-1}$, respectively [28] whereas pure tungsten does not display any optical Raman active mode.

\subsection{Laser-light-sheet scattering method}

The NP cloud dynamics was studied using the laserlight-sheet scattering method $[29,30]$. A vertical laser sheet of $2 \mathrm{~cm}$ height $\left(40 \mathrm{~mW}\right.$, wavelength $\lambda_{L}=$ $532 \mathrm{~nm}$, linear polarisation at $45^{\circ}$ with respect to the horizontal plane (i.e. scattering plane)) was passing through the electrode assembly in the gap between the half-cylinders (see Fig.1). The light scattered by 
the dust particles was recorded at $90^{\circ}$ by a 12 bits megapixel resolution digital CCD camera (BASLER ACE acA1300-60gm). A low frame rate of 1 frame per second and a long exposure time of $0.5 \mathrm{~s}$ were necessary to compensate for the low intensity of the scattered light. An interference filter centred on the laser wavelength was placed in front of the camera lens to reject most of the plasma emission. The position of the center of the laser sheet was adjusted between $1 \mathrm{~cm}$ and $9 \mathrm{~cm}$ above the anode during the experiments.

\subsection{Light extinction spectrometry (LES)}

\subsubsection{LES principle}

$N$ and the normalized PSD, $n$, were recovered in-line using the LES technique, which consists in analysing the spectral transmittance $T(\lambda)$ of a broadband and collimated light beam passing through the plasma containing the studied NP cloud. If $I_{0}$ and $I_{t}$ are the spectral intensities of the incident and the exiting beam respectively, $T(\lambda)$ can be defined as follows:

$T(\lambda)=\frac{I_{t}(\lambda)-I_{\text {plasma }}(\lambda)-I_{b}(\lambda)}{I_{0}(\lambda)-I_{b}(\lambda)}$

where $\lambda$ stands for the considered wavelength, $I_{\text {plasma }}$ for the intensity of the plasma emission in the direction of propagation of the incident beam and $I_{b}$ for the noise of the detection system (i.e dark noise and residual background optical noise). If the detection of multiple scattered photons is negligible, the measured transmission is simply an exponentially decreasing function whose argument is the product of the probing length $L$ (i.e the cloud width), and the cloud turbidity $\tau$. The latter is the product of $N$ and the mean extinction cross section $\overline{C_{e x t}}$ of the particles illuminated by the probing beam. $\overline{C_{\text {ext }}}$ is an integral quantity that depends on the extinction cross section of each particle (shape, size, refractive index, etc.) and its statistical weight in the particulate medium. It is more convenient to write the LES transmission equation in its linearised and discretised form [25]:

$\ln \left(T\left(\lambda_{i}\right)\right)=-N L \sum_{j=1}^{M_{D}} C_{i j} n_{j}$

where $C_{i j}$ is the statistical averaged extinction crosssection for the particle size class $j$ (with $j=$ $1,2, \ldots M_{D}$, where $M_{D}$ is the maximum number of considered size classes) and for the wavelength $\lambda_{i}$ (with $i=1,2, \ldots K_{i}$, where $K_{i}$ is the maximum number of considered wavelengths); $n_{j}$ is the probability density associated with the particle-size class centred on the diameter $D_{j}$.

The particle shape model used in the present study to describe tungsten NPs is a sphere (see section 4.1 for validation). Consequently, all the elements of $C_{i j}$ can be calculated using the Lorenz-Mie theory [30]. Particle inhomogeneities, such as porosity or tungsten oxide spherical inclusions, can also be taken into account using the Maxwell-Garnett effective media approximation. More details and comparisons with other light scattering theories can be found in Refs.[30, 25]. To illustrate the effects of particle inhomogeneities, the evolution of $C_{\text {ext }}$ is plotted in Fig.2 for pure (tungsten mass fraction, $f_{W}=100 \%$ ), porous ( $f_{W}=74 \%$, the remaining being empty) and oxidized $\left(f_{W}=74 \%\right.$ and $\left.f_{W O 3}=26 \%\right)$ tungsten NPs. The differences among the cases are mostly observed in the UV-range, where the size parameter $(\pi D / \lambda)$ of these Rayleigh-like particles is maximum, as well as the imaginary part of their refractive index [31].

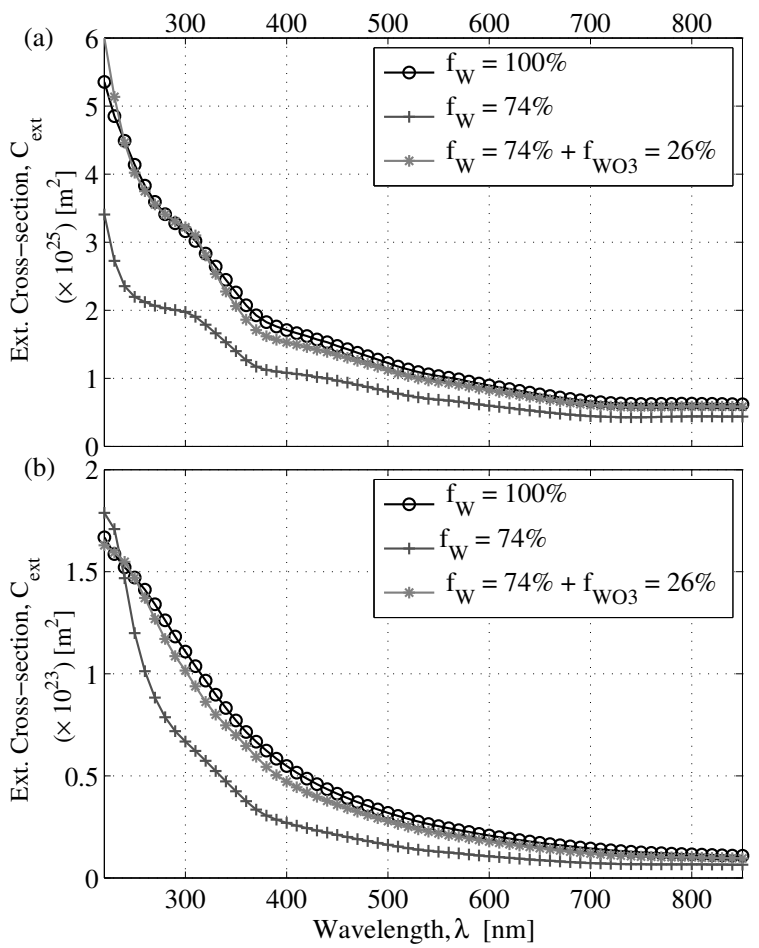

Figure 2. Spectral evolution of the extinction coefficient $C_{\text {ext }}$ of spherical particles of (a) $D=29 \mathrm{~nm}$, (b) $D=65 \mathrm{~nm}$ and of various compositions: pure tungsten, tungsten with spherical vacuum inclusions $\left(f_{W}=74 \%\right)$, tungsten with tungsten oxide spherical inclusions $\left(f_{W}=74 \%, f_{W O 3}=26 \%\right)$.

\subsubsection{Inversion procedure of LES measurements}

Solving Eq.2 requires to solve a discrete ill-posed problem $[31,25]$ and thus, to implement in the inversion algorithm a specific regularization scheme. The classical regularization methods, such as the PhilipsTwomey and Tikhonov methods or the truncated singular value decomposition, did not work properly when applied to the present experimental data. It mainly resulted from the weakness of the signal-to-noise ratio of 
experimental transmission spectra because of the relative low value of $N$ in the discharge. In the present study, to get more robust inversions, additional physical inputs were required. In Refs.[22, 24], it was shown that up to $500 \mathrm{~s}$, PSDs were mono-modal or bi-modal depending on the collection method. Based on these SEM analyses, PSDs were thus assumed to be of a particular type: unimodal or bimodal one. A mode $n(D)$ was simply described by a log-normal distribution depending of two parameters: a mean diameter $\bar{D}$ and a standard-deviation $\sigma$. The mode boundaries were deduced by truncating the PSD at $1 / 1000$ of its global maximum [25]. It followed that, for a bimodal distribution (i.e. two distinct particle populations $n_{1}(D)$ and $n_{2}(D)$ ), five parameters needed to be estimated: two mean diameters $\overline{D_{1}}$ and $\overline{D_{2}}$, two standard deviations $\sigma_{1}$ and $\sigma_{2}$, and a constant $\alpha$ quantifying the relative weight in number of each distribution: $n(D)=\alpha n_{1}(D)+(1-\alpha) n_{2}(D)$, with $0 \leq \alpha \leq 1$. All of these parameters could be deduced from a classical [25] iterative and weighted least squares algorithm that compares experimental and the theoretical extinction spectra (i.e. Eq. 2).

\subsubsection{Sensitivity of the LES inversion procedure}

In this section, numerical results providing physical insights and estimations of the LES technique resolution are reported. Five test cases, either unimodal or bimodal, are considered. Their statistical parameters and the associated logarithm of the synthetic transmittance spectra, $\ln (\mathrm{T})$, are reported in Table 1 and Fig.3a respectively. These spectra have been calculated for pure tungsten particles using the Lorenz-Mie theory. To focus on the effects of the particle size on the transmittance spectra, they were all calculated for a constant particle number concentration.

For synthetic unimodal populations (see case (a) and case (b), Table 1), an increase of the particle size leads to a decrease in transmistance, in particular at short wavelengths. In the case of a synthetic bimodal particle clouds, the shape of $\ln (\mathrm{T})$ spectra still depends on the size of the particles but also on the proportion $\alpha$ of a population with respect to the other one. A low proportion of small particles $(\alpha \leq 0.1)$ changes only slightly transmission rate at short wavelengths, as show the spectra associated with the cases (b) and (c) (Table 1). Larger proportion of small particles in the synthetic cloud $(\alpha \gtrsim 0.9)$ leads to an increase of the transmission rate (see spectra associated with the cases (d) and (e)).

To further test the capability of LES inversion procedure to distinguish between one or two particle populations, a white noise with an amplitude corresponding

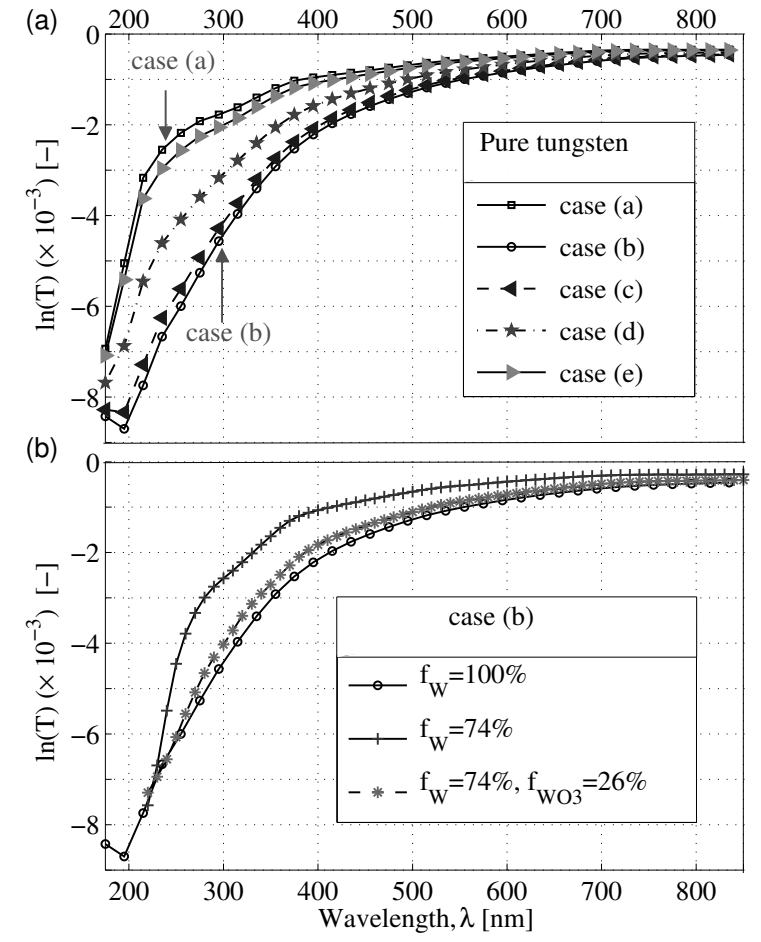

Figure 3. a) Evolution of the logarithm of synthetic transmittance spectra calculated for tugnsten spherical particles. The associated PSD parameters are reported in Table 1. b) Evolution of synthetic $\ln (\mathrm{T})$ spectra calculated in the case of an unimodal population (case (b) in Table 1) for pure, porous or oxidized tungsten NPs.

to $4 \%$ (i.e. $S N R \approx 14 d B$ ) of the minimum transmission was added to the transmittance spectra shown in Fig. 3a. Note that this noise amplitude is representative of the experimental one. The error between the recovered (after inversion) and the nominal statistical parameters of these synthetic PSDs are reported on Table 2. With an inversion of the first type (i.e researching unimodal PSDs), the statistical parameters of cases (a) and (b) are perfectly recovered, while those of bimodal populations (cases (c) ... (e)) are naturally poorly recovered. Note that for low values of $\alpha$, using a unimodal assumption leads to rather satisfactory estimation of $\overline{D_{2}}$ and $\sigma_{2}$, i.e the particle population with the largest size and highest concentration. This is consistent with the previous observation regarding spectra of bimodal particle clouds containing only a low concentration of small particles. On the contrary, using a second type inversion (i.e researching bimodal PSDs) allows a good reconstruction of all of these synthetic PSDs (see Table 2).

LES measurements and inversions also depend on the particle composition (through the particle complex refractive index). As an illustration, Table 3 reports the errors between the parameters of the recovered and the nominal PSDs in the case of an unimodal distribu- 
Table 1. Parameters of synthetic PSDs associated with spectra of Fig.3. $\overline{D_{m}}$ and $\sigma_{m}$ are the statistical parameters of the unimodal populations equivalent to the bimodal populations (they are obviously unchanged for unimodal populations).

\begin{tabular}{cccccccc}
\hline \multicolumn{7}{c}{ Dependent model: nominal parameters } \\
\hline Cases & $\overline{D_{1}}$ & $\sigma_{1}$ & $\overline{D_{2}}$ & $\sigma_{2}$ & $\alpha$ & $\overline{D_{m}}$ & $\sigma_{m}$ \\
{$[\mathrm{~nm}]$} & {$[\mathrm{nm}]$} & {$[\mathrm{nm}]$} & {$[\mathrm{nm}]$} & $\begin{array}{c}\left.{ }^{2}\right] \\
{[\mathrm{nm}]}\end{array}$ & $\begin{array}{c}\text { Unimodal cases } \\
{[\mathrm{nm}]}\end{array}$ \\
\hline case (a) & 29 & 5 & 0 & 0 & 1 & 29 & 5 \\
case (b) & 65 & 5 & 0 & 0 & 1 & 65 & 5 \\
\hline \multicolumn{7}{c}{ Bimodal cases } \\
\hline case (c) & 29 & 5 & 65 & 5 & 0.1 & 61.4 & 11.8 \\
case (d) & 29 & 5 & 65 & 5 & 0.5 & 47.1 & 18.6 \\
case (e) & 29 & 5 & 65 & 5 & 0.9 & 32.7 & 11.7 \\
\hline
\end{tabular}

Table 2. Error ("Err") between the recovered and nominal mean diameters and standard deviations of synthetic spectra of Fig.3a. A negative error indicates an underestimation of the statistical parameter. In cases (c - e), for unimodal inversion method, the error between recovered and nominal parameters are calculated using the mean values: $\overline{D_{m}}$, and $\overline{\sigma_{m}}$.

\begin{tabular}{crrrcccc}
\hline \multicolumn{7}{c}{ Unimodal } & \multicolumn{5}{c}{ Bimodal } \\
\hline Cases & $\begin{array}{r}\operatorname{Err}(\bar{D}) \\
{[\mathrm{nm}]}\end{array}$ & $\begin{array}{c}\operatorname{Err}(\sigma) \\
{[\mathrm{nm}]}\end{array}$ & $\begin{array}{c}\operatorname{Err}\left(\overline{D_{1}}\right) \\
{[\mathrm{nm}]}\end{array}$ & $\begin{array}{c}\operatorname{Err}\left(\sigma_{1}\right) \\
{[\mathrm{nm}]}\end{array}$ & $\begin{array}{c}\operatorname{Err}\left(\overline{D_{2}}\right) \\
{[\mathrm{nm}]}\end{array}$ & $\begin{array}{c}\operatorname{Err}\left(\sigma_{2}\right) \\
{[\mathrm{nm}]}\end{array}$ & $\begin{array}{c}\operatorname{Err}(\alpha) \\
{[-]}\end{array}$ \\
\hline case $(\mathrm{a})$ & 2.0 & 0.0 & 2.0 & 0.0 & - & - & 0.0 \\
case (b) & -1.0 & -1.0 & -1.0 & -1.0 & - & - & 0.0 \\
case (c) & 0.6 & -4.8 & 2.0 & 0.0 & -1.0 & -1.0 & 0.0 \\
case (d) & 1.9 & -5.6 & 2.0 & 0.0 & -1.0 & -1.0 & 0.0 \\
case (e) & -0.7 & 1.3 & 2.0 & 0.0 & -1.0 & -1.0 & 0.0 \\
\hline
\end{tabular}

Table 3. Error between the recovered and nominal mean diameters and standard deviations of synthetic spectra of Fig.3b.

\begin{tabular}{cccccrr}
\hline Assumed compositions & \multicolumn{2}{c}{ Pure } & \multicolumn{2}{c}{ Porous } & \multicolumn{2}{c}{ Oxidized } \\
\hline & $\begin{array}{c}\operatorname{Err}(\bar{D}) \\
{[\mathrm{nm}]}\end{array}$ & $\begin{array}{c}\operatorname{Err}(\sigma) \\
{[\mathrm{nm}]}\end{array}$ & $\begin{array}{c}\operatorname{Err}(\bar{D}) \\
{[\mathrm{nm}]}\end{array}$ & $\begin{array}{c}\operatorname{Err}(\sigma) \\
{[\mathrm{nm}]}\end{array}$ & $\begin{array}{r}\operatorname{Err}(\bar{D}) \\
{[\mathrm{nm}]}\end{array}$ & $\begin{array}{r}\operatorname{Err}(\sigma) \\
{[\mathrm{nm}]}\end{array}$ \\
\hline Pure, $f_{W}=100 \%$ & -1.0 & -1.0 & -2.0 & 20.0 & 5.0 & 0.0 \\
Porous, $f_{W}=74 \%$ & -9.0 & -4.0 & -1.0 & -1.0 & -20.0 & 0.0 \\
Oxidized, $f_{W}=74 \%$ & -8.0 & -4.0 & -1.0 & 13.1 & -1.0 & -1.0 \\
\hline
\end{tabular}

tion (case (b), Table 1): the tungsten particles were alternatively pure (tungsten mass fraction, $f_{W}=100 \%$ ), porous $\left(f_{W}=74 \%\right)$ or partly oxidized $\left(f_{W}=74 \%\right.$ and $\left.f_{W O_{3}}=26 \%\right)$. As mentioned above, for these calculations, the Maxwell-Garnett effective medium approximation was used to account for the effects of pores or $\mathrm{WO}_{3}$ inclusions. As before, a white noise with a relative amplitude of $4 \%$ is added on the associated $\ln (\mathrm{T})$ spectra. It turns out that, globally, an overestimation of the particle density leads to an underestimation of the mean diameter and to a slight underestimation of the standard deviation. $\mathrm{WO}_{3}$ inclusions do not have a large effect on the synthetic $\ln (\mathrm{T})$ spectra.

In Fig.4, the recovered evolution of the mean diameter as a function of the relative noise amplitude of the synthetic transmission rate corresponding to the PSD of case (b) is plotted. When the relative amplitude is lower than $4 \%$, the statistical parameters of case (b) are almost perfectly reconstructed, which is not the case for higher relative noise amplitude. In particular, an increase of the noise amplitude leads to an overestimation of the value of $\sigma_{1}$. However, the error on $D_{1}$ does not exceed $15 \%$ even for noise amplitude $\lesssim 12 \%$. The absolute error on $N$ also increases with the noise amplitude but the recovered values are within $30 \%$ of the real one for noise amplitude $\lesssim 12 \%$.

Given all the numerical tests, it can be concluded that, on the conditions that one has a minimum knowledge on the NPs (shape, composition) and makes reasonable assumptions on the PSD shape, LES is an accurate diagnostic even in noisy environments.

\subsubsection{LES Experimental setup}

The LES set-up (See Appendix A) was composed of a highly-stabilized Halogen-Deuterium lamp (Ocean Optics, DH-2000-DUV), solarisation resistant optical fibres with a $100 \mu \mathrm{m}$ core, an on-line intensity attenuator, achromatic coupling and collimating optics (50 $\mathrm{nm}$ focal length parabolic mirrors), a diaphragm 

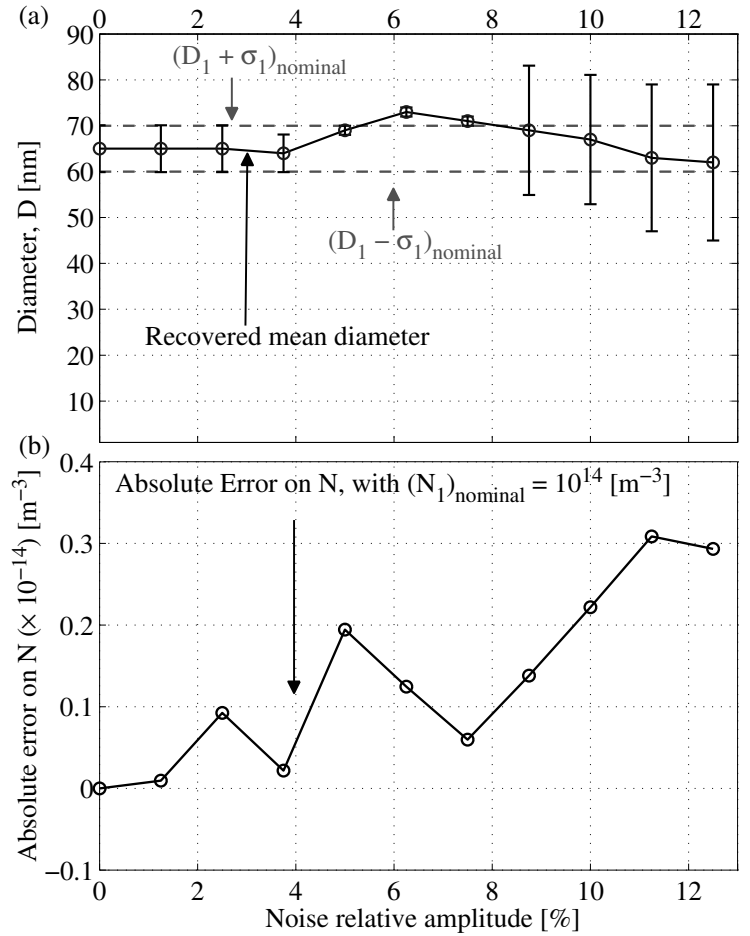

Figure 4. (a) Evolution of the recovered first two moments of the PSDs as a function of the relative noise amplitude. (b) Evolution of the absolute error on $N$ as a function of the relative noise amplitude. Synthetic spectra are calculated using the PSD of case (b) (see Table 1).

and a CCD spectrometer (Oceans Optics, Maya Pro). This low noise and high dynamic range spectrometer has an enhanced response in the UV range and a global half-height resolution of $2-5 \mathrm{~nm}$ in the spectral range 220-1040 $\mathrm{nm}$ where LES measurements were performed. Full optical calculations have demonstrated that for the considered particle size and concentrations, and because of the small aperture angle of the LES detection system (less than $1^{\circ}$ ), multiple scattering is negligible. Optical choppers were used to chop the probing beam of $1 \mathrm{~mm}$ in diameter, in order to record periodically: the optical and electronic signals, the reference signal and the plasma light emissions. This allowed us to record one corrected transmission spectrum (see Eq.2) every $2 \mathrm{~s}$.

\section{Results}

\subsection{Electron microscopy}

SEM results have been described in details in previous articles $[22,24]$. Here is a quick outline of the main results obtained:

- NPs were pushed towards the anode located at the bottom of the discharge set-up and could get detrapped and fall on the anode.
- The later the substrate was exposed to the plasma, the bigger were the collected NPs. The biggest NPs could have diameter up to $150 \mathrm{~nm}$ for discharge duration of $500 \mathrm{~s}$.

- Small particles (mean diameter $\sim 30 \mathrm{~nm}$ ) were always observed on the substrate when the discharge was switched off (method (ii), see section 3.1). These results indicated constant nucleation and growth of NPs in the upper part of the discharge (cathode side).

Electron microscopy studies of particles collected after a $300 \mathrm{~s}$ plasma indicate that NPs are roughly spherical, and look mainly like a compact aggregate of tungsten crystallites ( $d \simeq 2-4 \mathrm{~nm}$ ) (see Fig.5), justifying partially our hypothesizes on the particle shape model for LES data inversion (see Fig.7 in Ref. [25]).

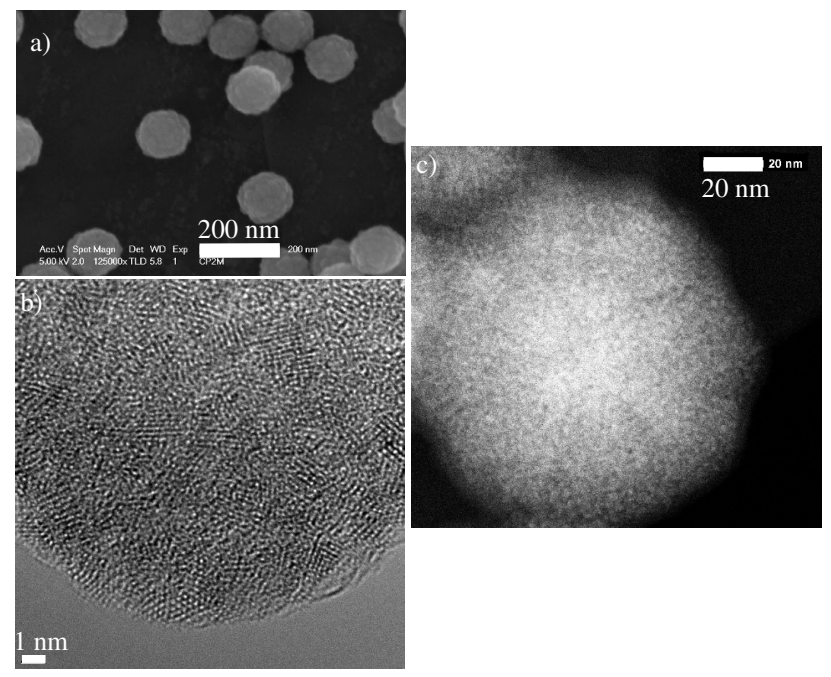

Figure 5. Images of a NP collected after a $300 \mathrm{~s}$ plasma. a) SEM. b) HR-TEM. c)HAADF-STEM. The fringe patterns in b) are the crystallites. The white dots in c) are the tungsten crystallites.

\subsection{Raman spectroscopy}

Measurements were performed directly on the cathode and on stainless steel samples covered with NPs. Weak tungsten oxide signatures were observed by Raman spectroscopy. In order to check if NPs were weakly oxidised or not, they have been heated under an oxygen containing atmosphere. The growth of tungsten oxide bending and stretching modes was observed only for temperature higher than $700^{\circ} \mathrm{C}$. Final Raman scattering intensities were multiplied by a factor of $\sim 25$ showing that the weak bands, observed at room temperature were due to surface oxide, probably formed during the venting of the chamber, after the experiments. It is worth reminding that we have shown in Sec.3.4.3 that 
a small amount of oxide in the NPs has a negligible impact on LES transmittance spectra. Therefore, for the rest of this study, it was assumed that the NPs in the plasma were composed of tungsten crystallites and, consequently, complex refractive index of pure tungsten [32] was used to inverse LES measurements.

\section{3. laser-light-sheet scattering measurements}

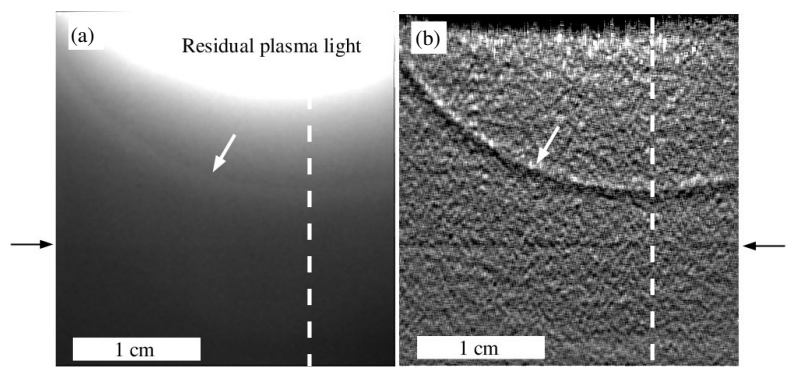

Figure 6. Typical snapshot of the scattered laser-light $45 \mathrm{~s}$ after plasma ignition : a) raw image, b) same as a) with an edge detection filter applied to the image. The horizontal black arrow shows the position of the lower edge of the laser sheet which was positioned $2 \mathrm{~cm}$ below the cathode. The oblique white arrow shows the top edge of the NP cloud. The dashed vertical line indicates the position of the discharge axis.

In an earlier study [24], laser-light-sheet scattering measurements just above the anode revealed that the dust particle cloud was compressed and pushed towards the anode during the discharge. For a better understanding of the dynamics of the growing process, further experiments with different positions of the laser-light sheet above the anode have been performed. In each experiment, no NPs could be detected by our laser-light scattering system during the first $30 \mathrm{~s}$ after plasma ignition.

In Fig.6a, a snapshot of the scattered laser-light $45 \mathrm{~s}$ after plasma ignition is shown. The centre of the laser-light-sheet was positioned at $2 \mathrm{~cm}$ of the cathode surface (i.e. $8 \mathrm{~cm}$ from the anode). For a better visualisation of the boundary of the dust cloud, an edge detection was applied to the raw image (Fig.6b). The top edge of the cloud had a very convex shape and an apparently dust-free hole centred on the discharge axis was visible (no NP detected by laser-light scattering). In Fig.6a, one can see that there was a strong plasma emission in this region. It corresponded to the expanding luminous "blue glow" appearing at the centre of the tungsten cathode a few seconds after plasma ignition [22]. During the hemispherical expansion of this glow, the NP cloud was pushed toward the bottom and the side of the discharge gap explaining its convex shape.

In Fig.7a, three snapshots are shown for a laserlight sheet positioned $3.4 \mathrm{~cm}$ above the anode. The
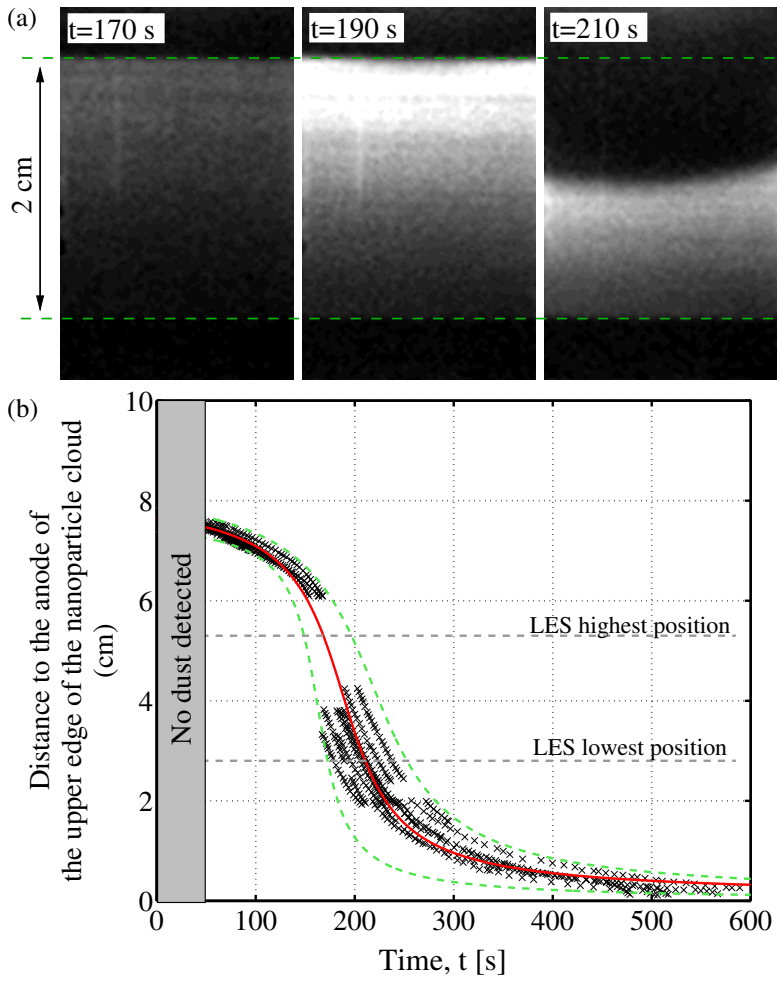

Figure 7. (colour online) a) Snapshots of the video of the scattered laser-light at different times after plasma ignition. The dashed green lines show the upper and lower edges of the vertical laser sheet. The centre of the laser sheet was $3.4 \mathrm{~cm}$ above the anode. b) Distance of the upper edge of the NP cloud ( $1^{\text {st }}$ generation) to the anode on the discharge axis as a function of time extracted from laser-light scattering data. The latter were accumulated over 21 experiments. The red line represents a fitted mean position of the upper edge of the NP cloud. The dashed green lines are the errorbars at 3 standard deviations.

scattered light intensity increased with time until the dust cloud was pushed down and toward the side of the discharge and, as observed close to the cathode, an apparently "dust-free" hole was expanding in the discharge. This behaviour was observed at all investigated heights between the electrodes and, as already stated, could be linked to the expanding luminous "blue glow" observed on the cathode centre a few second after plasma ignition (see Fig.6) [22]. This effect was clear at $t=210 \mathrm{~s}$ after plasma ignition: in Fig.7a the top edge of the NP cloud is convex. Note that the intensity gradients observed in Fig. 7 are the result of a convolution between the vertical intensity profile of the laser-light sheet, with the NP size/number concentration gradients in the region illuminated by the laser-light sheet. Since the scattering regime for the considered NPs is in between the Rayleigh regime and the lower bound of the Mie scattering regime, the NP scattering efficiency is proportional to $D^{6}$ for the smallest and tends to (but does not reach) the limit of $D^{2}$ for the 
largest NPs. That is any detailed interpretation of the continuous changes in the intensity gradients (or "inhomogeneities") observed in Fig. 7a, has to be done with caution.

In Fig.7b, the distance of the upper edge of the NP cloud is plotted as a function of time. Note that even though every experiment was carried out with the same discharge parameters, there was a strong dispersion in the measured time $( \pm 50 \mathrm{~s}$ for the cloud top edge positioned at $3 \mathrm{~cm}$ above the anode). This is due to the high sensitivity of NP growth to the initial conditions (base pressure, cathode surface state, etc.). This effect was also observed in radio-frequency discharge in which NPs are grown by sputtering of a polymer layer on the electrode [33]. The general behaviour of the NP cloud was however always the same. The maximum height of the dust cloud edge was $\sim 7.5 \mathrm{~cm}$ above the anode at $\mathrm{t} \simeq 30 \mathrm{~s}$ after plasma ignition. Soon after the NP cloud could be detected, it started to be pushed toward the anode and the edge of the discharge. For $\sim 100 \mathrm{~s}$, the movement of the NP cloud was quite slow and the height variation of the upper edge was $\sim 1 \mathrm{~cm}$. Then, in $\sim 200$ s the upper edge position felt from $\sim 6.5 \mathrm{~cm}$ to less than $1 \mathrm{~cm}$, meaning that a major part of the plasma seemed free of dust. However, our previous studies have shown that small NPs (hardly detectable by laser-light scattering ) were growing in this region [24].

\subsection{Light extinction spectrometry}

In Fig.8a, the temporal evolution of the transmittance at different wavelengths is shown when the probing beam was positioned $2.8 \mathrm{~cm}$ above the anode. As expected for NPs, the transmittance at short wavelengths was more affected by the presence of the growing tungsten NPs than at long wavelengths (i.e. $T \simeq 1$ ). In Fig.8b, evolutions of the transmittance at $\lambda=250 \mathrm{~nm}$ for five experiments with the same discharge parameters (two measurements at $h=5.3 \mathrm{~cm}$ and three measurements at $h=2.8 \mathrm{~cm}$ ) are shown. While not exactly the same, due to the high sensitivity of the experiments to the initial conditions, one can see that the measurements at the same height were similar. The overall shape was comparable for both heights but the kinetics was faster in the measurements made at the highest positions. For instance, the minimum of transmittance occurred at $t \simeq 170 \mathrm{~s}$ at $h=5.3 \mathrm{~cm}$ while it occurred at $\mathrm{t} \simeq 220 \mathrm{~s}$ at $h=$ $2.8 \mathrm{~cm}$. These minima in transmittance could be linked to the opening of the apparently "dust-free" hole at the investigated height. The times of the minima matched indeed within errorbars the position of the upper edge of the dust particle cloud on the discharge axis (see Fig.7b). Before reaching a transmission minimum, the NP number concentration and/or NP
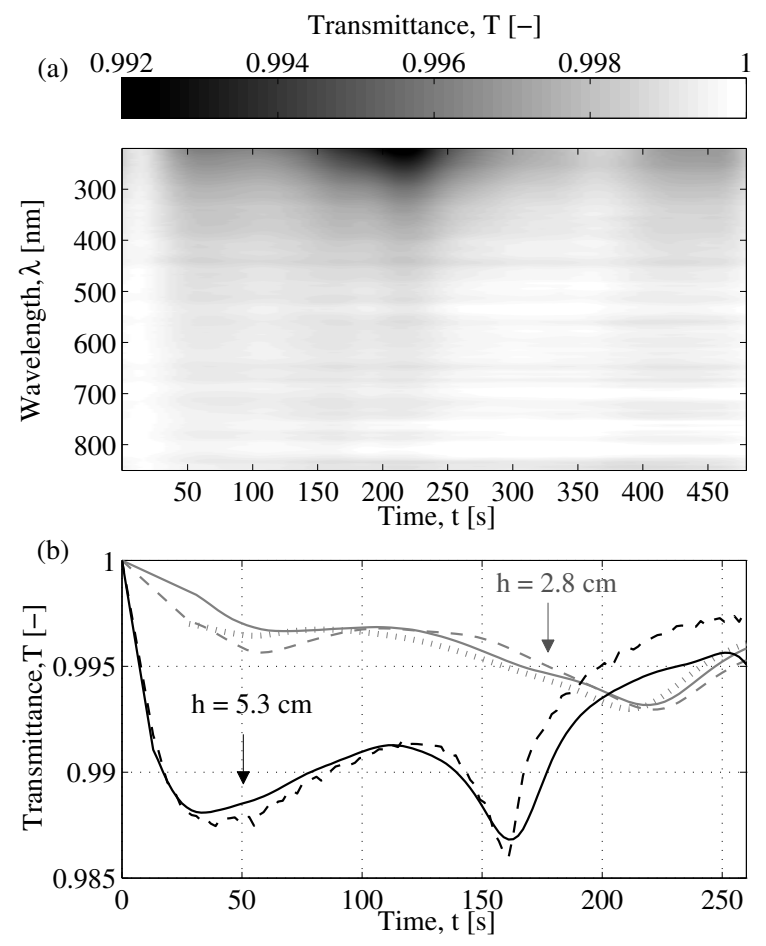

Figure 8. a) Evolution of the transmitance at different wavelengths as a function of time, $2.8 \mathrm{~cm}$ above the anode for a $500 \mathrm{~s}$ discharge. b) Transmittance at $250 \mathrm{~nm}$ at different heights above the anode: gray lines, $h=2.8 \mathrm{~cm}$; black lines, $h=5.3 \mathrm{~cm}$. Each plain, dashed, or dotted line corresponds to a separate experiment.

size in the LES beam have increased which explained the transmittance decrease. From this time, the path length of the LES beam through the cloud visible by laser-light-sheet scattering started to decrease and the transmittance started to increase. Note that it was previously reported [24] that small NPs were present in this hole. Extinction being very sensitive to the NP size, the larger having the greatest effect, replacing large NPs by smaller one is almost similar in effect to a dust-free zone.

It may appear surprising that LES transmittances at higher probing positions in the plasma were smaller than at lower LES positions. However, as the cloud was pushed toward the side of the discharge, some particles were constantly lost through the vertical slits between the half glass tubes used for the optical diagnostics, explaining the higher transmittance at lower beam positions.

In Figs.9a and b, the recovered evolution of the PSD and $N$ are presented. They correspond to the transmittance spectrum shown in Fig.8a. Six different phases could be extracted in the measured NP growth and dynamics $\S$.

$\S$ Note that these phases occurred independently of the LES scanning position, the kinetics at the highest position being only 

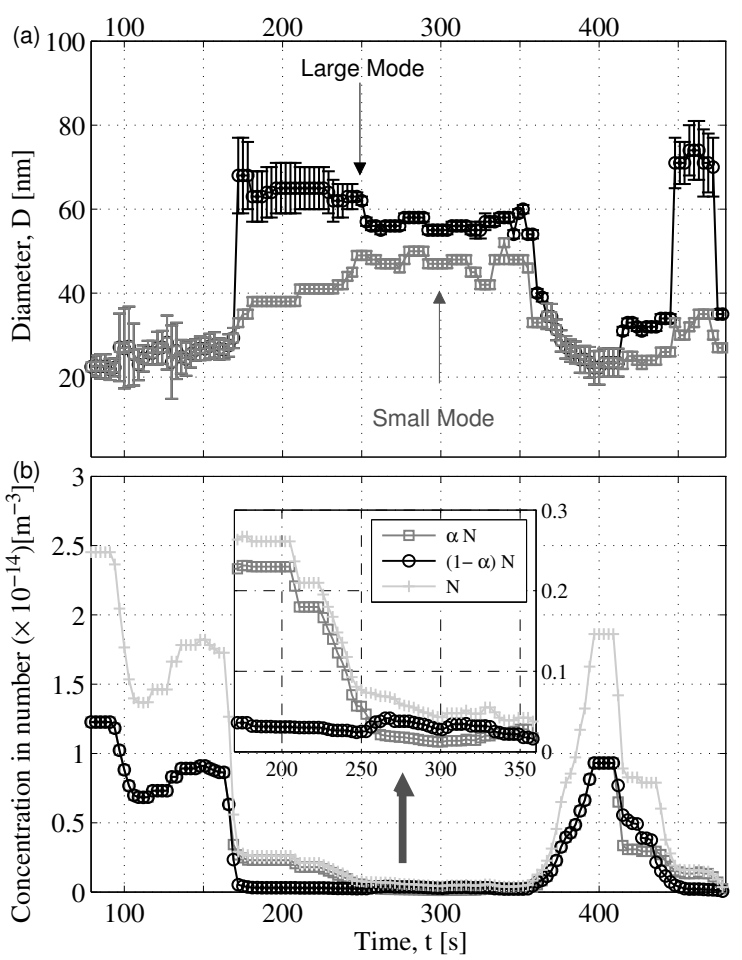

Figure 9. a) Temporal evolution of mean diameters (represented by squares or by circles) and the associated standard-deviations (represented by bars) measured by LES at the height $h=2.8 \mathrm{~cm}$ from the anode. A bimodal PSD is detected, its large mode is represented by the circle line, while its small mode is represented by the square line. b) Temporal evolution of $N$, as well as $\alpha N$ and $(1-\alpha) N$ associated with the small and large modes of the PSD, respectively.

- Before $\sim 70 \mathrm{~s}$, no information could be accurately extracted from the transmittance spectra. It indicates that NPS in the LES beam were very small and possibly in very low number concentration.

- From $\sim 70 \mathrm{~s}$ to $\sim 170 \mathrm{~s}$, the PSD was found to be clearly unimodal, as the inversion procedure forcing a bimodal distribution gave two identical modes with the same weight. During this phase, the mean particle diameter increased from $\sim 20 \mathrm{~nm}$ to $\sim 30 \mathrm{~nm}$. $N$ was nearly constant $\left(\sim 2 \cdot 10^{14} \mathrm{~m}^{-3}\right)$ even if a smooth decrease (over $\sim 12 \mathrm{~s}$ ) in $N$, coinciding with higher values of $\sigma_{1}$, was observed at $t \sim 80 \mathrm{~s}$. This increase in $\sigma_{1}$ could be attributed to local higher values of the relative noise in LES measurements (see results of the synthetic study presented in section 3.4.3, more particularly in Fig. 4) that could be due to very intense and fluctuating plasma emissions occuring after ignition [22]. As a transmittance spectrum depends on a convolution between $N$ and the PSD, large recovered values of $\sigma_{1}$ (i.e. a large distribution) imply small recovered values of $N$. Moreover, the PSD remained mono-modal after 80 s which favours the hypothesis of an inversion artefact.

- At $t \sim 170 \mathrm{~s}$, an inflection point is observed on the transmittance rate at all wavelengths, while the PSD became abruptly bi-modal: each mode which were initially identical are suddenly well separated. Simultaneously, the total concentration in number decreased abruptly (in less than $\sim 6 \mathrm{~s}$ ). The number concentration of the small (i.e original) mode particles decreased also abruptly, while the concentration of the large mode particles, which just appeared, was very small. These observations suggest an agglomeration of the small particles at constant volume. Nevertheless as the agglomeration process was not instantaneous, small particles were not totally consumed and some remained in the LES beam. After this time, the mean diameter of the particles in the large mode stayed almost constant while the mean diameter of the particles of the small mode steadily increased.

Two mechanisms could explain the apparent growth of the NPs of the small mode: surface growth by molecular sticking or agglomeration. If the NPs of the small mode were growing by surface deposition or by agglomeration with very small undetectable particles, their number concentration should be constant. It was not the case here, Fig. 9 clearly shows a decrease of their number concentration. In addition, it can be shown using the theory of Ref.[34], that tungsten vapour concentration is maximum around $1 \mathrm{~cm}$ below the cathode and is rapidly decreasing when going towards the anode. Consequently, surface growth on the anode side, where LES measurements were performed, was most probably negligible.

A more likely explanation is that they were consummed by agglomeration to create the NPs of the large mode. Even if the agglomeration of the $35 \mathrm{~nm}$ particles should normally be suppressed because they are negatively charged, it may still exist due to a mechanism called the polarizationinduced ion flux asymmetry (PIFA) [35, 36]. In the PIFA mechanism, it is proposed that a NP becomes polarised due to the influence of the electric field created by the neighbouring grain which leads to an asymmetry of the ion flux onto the surface grain resulting in a force in the direction of the electric field. PIFA mechanism thus allows negatively charged large particle to agglomerate with smaller negatively charges one. It was verified experimentally in a $\mathrm{RF}$ discharge 
[36]. Furthermore, as NPs were getting deeper in the negative glow, i.e. closer to the anode, the electron temperature decreased and so did the NP charge (in absolute value) reducing the repulsive force.

Finally, the agglomeration hypothesis is favoured by the sudden appearance of the large mode in concordance with a sudden decrease of the number concentration of the NPs of the small mode. One should also note that the two modes of the PSD are log-normal and only the mean sizes are given in Fig.9. While the PIFA mechanism allows the agglomeration of negatively charged particles, it still favours the agglomeration of the smallest one with the largest one. Consequently, smallest NPs were consumed in priority and, in return, the mean diameter of the small mode increased. Experiments at different heights showed that this phase occurs quicker when closer to the cathode. A possible explanation for the triggering of the agglomeration phase is that when large particle pass through a cloud of smaller particle, agglomeration is enhanced [37, 35, 36].

- At $\sim 220 \mathrm{~s}$, the transmittance reached a minima at all wavelengths and from this time $N$ started to decrease rapidly. When looking at the two modes of the PSD, their mean size remained almost unchanged but the weight of the smaller particles decreased rapidly. As discussed previously, this corresponded to the opening of the "dust-free" region in the LES beam. From this time, the path length of the LES beam through the cloud (visualised by laser-light-sheet scattering) started to decrease and the transmittance started to increase due to a decreasing number density. Note that in the region freed by the first generation of NPs, new NPs were starting to grow [24]. They however remained undetectable with the LES diagnostics as, extinction being very sensitive to NP size, large NPs had the greatest effect and the overall effects was almost similar to a "dustfree" zone. It also explains why the weight of smaller mode of the PSD was more affected. As stated earlier, the evolution of the LES signal was similar for the different investigated positions of the probing beam. Only the dynamics was faster when closer to the cathode. After inversion of the LES spectra, another difference appeared. In Fig.10, the size of the particle at the minimum of transmittance for different LES beam height are presented. The PSD was bimodal at all investigated heights. No trend is observable for the mean size of the NPs belonging to the small mode which remain around $40 \mathrm{~nm}$ while the mean size of the particle belonging to the large mode is increasing when getting closer to the anode. This is a clear indication for a continuous growth of NPs while getting transported towards the anode. Earlier measurements of the particle collected on the anode showed that the NPs can reach size up to $150 \mathrm{~nm}$ [24]. Such large size were not measured by LES, strongly suggesting that NP growth continued below the minimum investigated LES beam height. This is also compatible with the earlier hypothesis of an agglomeration phase enhanced by the penetration of large NPs into the cloud of smaller particles, the former growing further while transported towards the anode.

- From $\sim 250$ s to $\sim 350 \mathrm{~s}$ (see Fig. 9), the two modes of the PSD were almost superimposed ( $\sim 50 \mathrm{~nm}$ and $\sim 60 \mathrm{~nm})$. The number concentrations of both mode were equivalent $\left(\leq 0.1 \cdot 10^{14} \mathrm{~m}^{-3}\right)$ and the total number concentration was slowly decreasing. These observations were the consequence of two effects: the agglomeration process which consumed the smallest particle and the opening of the "dust-free" zone in the LES beam. However, it has to be noted that the two modes of the PSD remain distinct from each other as the largest particles of the original small mode are probably too big to agglomerate, even considering the PIFA mechanism.

- From $\sim 350$ s to $\sim 400$ s, $N$ increased rapidly while the mean NP diameter felt down to $\sim 35 \mathrm{~nm}$ (monomodal distribution). On the transmittance spectrum, it corresponded to a new decrease of the transmittance. This period of time corresponded to the expulsion of the last large NPs of the first generation and consequently, an easier detection of the second generation of NPs. It is in accordance with our earlier experiments [24]: large NPs were expelled from the plasma and new small ones were synthesized in the region freed from the first generation (mainly above as the NP cloud is pushed down) where nucleation could take place again from sputtered atoms. This behaviour is similar to the one observed in radio-frequency discharges where a "void" usually appears in the plasma center, in which new dust particles are grown [38, 33, 39].

- From $\sim 400 \mathrm{~s}$, the PSD became again bi-modal with an associated decrease of $N \mathrm{n}$ in a very similar manner to what happened at $t \sim 170 \mathrm{~s}$. These were signs of an agglomeration process occurring in the second generation NP cloud.

\section{Conclusion and perspectives}

In this article, a detailed characterisation of a growing NP cloud in a DC sputtering low pressure argon glow 


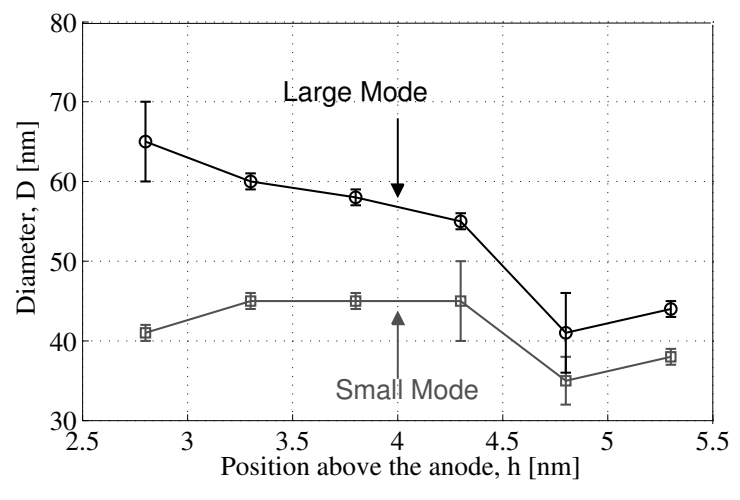

Figure 10. Evolution of the particle size reached at the minima in transmittance.

discharge with a tungsten cathode was performed. The shape and composition of NPs with ex-situ diagnostics (SEM,TEM.HAADF-TEM and Raman spectroscopy) was determined. The particles were found to be mainly compact spheroids of pure tungsten crystallites which allowed us to use the complex refractive index of pure tungsten for the LES diagnostics. Laserlight scattering of a vertical laser sheet positioned at different heights in the inter-electrode gap showed that the NP cloud was pushed towards the edge of the discharge and the anode leaving a zone in which nucleation of new NPs could take place. In addition, the evolution of the PSD and $N$ were studied in-situ by LES. This diagnostic has revealed a phase of NP growth by agglomeration, followed by the appearance of a second NP generation. The agglomeration phase was identified by a sudden change of the PSD from monomodal to bi-modal followed by a gradual disappearance of the small NPs. Results have also confirmed that the second generation of NPs appeared in the space freed by the first NP generation. At the minimum of transmittance, while the PSD was bi-modal at all investigated positions between the electrode, the mean size of the NPs belonging to the large mode of the PSD increased when getting closer to the anode. On the contrary, the mean size of the NPs belonging to the small mode remained approximately constant. This results suggests that agglomeration was triggered when the biggest NPs passed through a cloud of smaller NPs. They thus grew until they reach the anode side of the discharge. It shows that the largest particles are agglomerates of smaller $\sim 35 \mathrm{~nm}$ particles and is consistent with our results reported in earlier studies using electron microscopy measurements to reconstruct PSDs of particle collected at the anode centre $[22,23$, 24]. Furthermore, as the NP growth continued below the LES scanning positions, it explains the apparent dissimilarities observed between the in-situ optical and the ex-situ electron microscopy PSD measurements.
Future investigations will combine local measurements of plasma properties with the in-situ PSD diagnostics, to confirm some interpretations drawn in the present paper and to correlate NP growths and dynamics to the local evolution of the plasma parameters.

\section{Acknowledgments}

This work was supported by the INSIS of CNRS via PEPS (Projets Exploratoires Premier Soutien) grant. The authors would also like to thank S. Khrapak and the A*MIDEX foundation for their financial support in the framework of ANR-11-IDEX-001-02 and ANR11-LABX-0092 (Labex MEC). We are grateful to M. Cabié for her technical support in electron microscopy.

\section{Appendix A. LES set-up}

The LES set-up (See Fig. A1) was composed of a highly-stabilized Halogen-Deuterium lamp (Ocean Optics, DH-2000-DUV) (5), solarization resistant optical fibres (6) with a $100 \mu \mathrm{m}$ core, an on-line intensity attenuator (7), achromatic coupling and collimating optics (50 nm focal length parabolic mirrors) (8-9), a diaphragm (10) and a CCD spectrometer (Oceans Optics, Maya Pro) (12). Optical choppers (11a - 11b) were used to chop the probing beam of $1 \mathrm{~mm}$ in diameter and a typical probing length of $10 \mathrm{~cm}$, in order to record periodically: the optical and electronic signals, the reference signal and the plasma light emissions. This allowed us to record one corrected transmission spectrum (see Eq. 2) every $2 \mathrm{~s}$.

[1] Stefanovic I, Kovacevic E, Berndt J, Pendleton Y and Winter J 2005 Plasma Phys. Control. Fus. 47 A179

[2] Winter J and Gebauer G 1999 J. Nucl. Mat. 228 266-269

[3] Arnas C, Pardanaud C, Martin C, Roubin P, Temmerman G D and Counsell G 2010 J. Nucl. Mat. 401130

[4] Winter J 2004 Plasma Phys. Control. Fus. 46 B583

[5] Smirnov R D, Pigarov A Y, Rosenberg M, Krasheninnikov S I and Mendis D A 2007 Plasma Phys. Control. Fus. $49347-371$

[6] Arnas C, Martin C, Roubin P, Pégourié B, Temmerman G D, Hassouni K, Michau A, Lombardi G and Bonnin X 2010 Plasma Phys. Control. Fus. 52124007

[7] Boufendi L, Plain A, Blondeau J P, Bouchoule A, Laure C and Toogood M 1992 Appl. Phys. Lett 60169

[8] Plain A 1998 J. Appl. Phys. 834012

[9] Watanabe Y 2006 J. Phys. D: Appl. Phys. 39 R329

[10] Hollenstein C, Dorier J L, Dutta J, Sansonnens L and Howling A A 1994 Plasma Sources Sci. Technol. 3278

[11] Mikikian M, Couëdel L, Cavarroc M, Tessier Y and Boufendi L 2010 Phys. Rev. Lett. 105075002

[12] Graves D, Daugherty J, Kilgore M and Porteous R 1994 Plasma Sources Sci. Technol. 3433

[13] Stefanovic I, Berndt J, Maric D, Samara V, RadmilovicRadjenovic M, Petrovic Z L, Kovacevic E and Winter J 2006 Phys. Rev. E 74026406

[14] Selwyn G S 1994 Plasma Sources Sci. Technol. 3 340-347

[15] Hayashi Y and Tachibana K 1994 Jpn. J. Appl. Phys. 33 L804-L806 


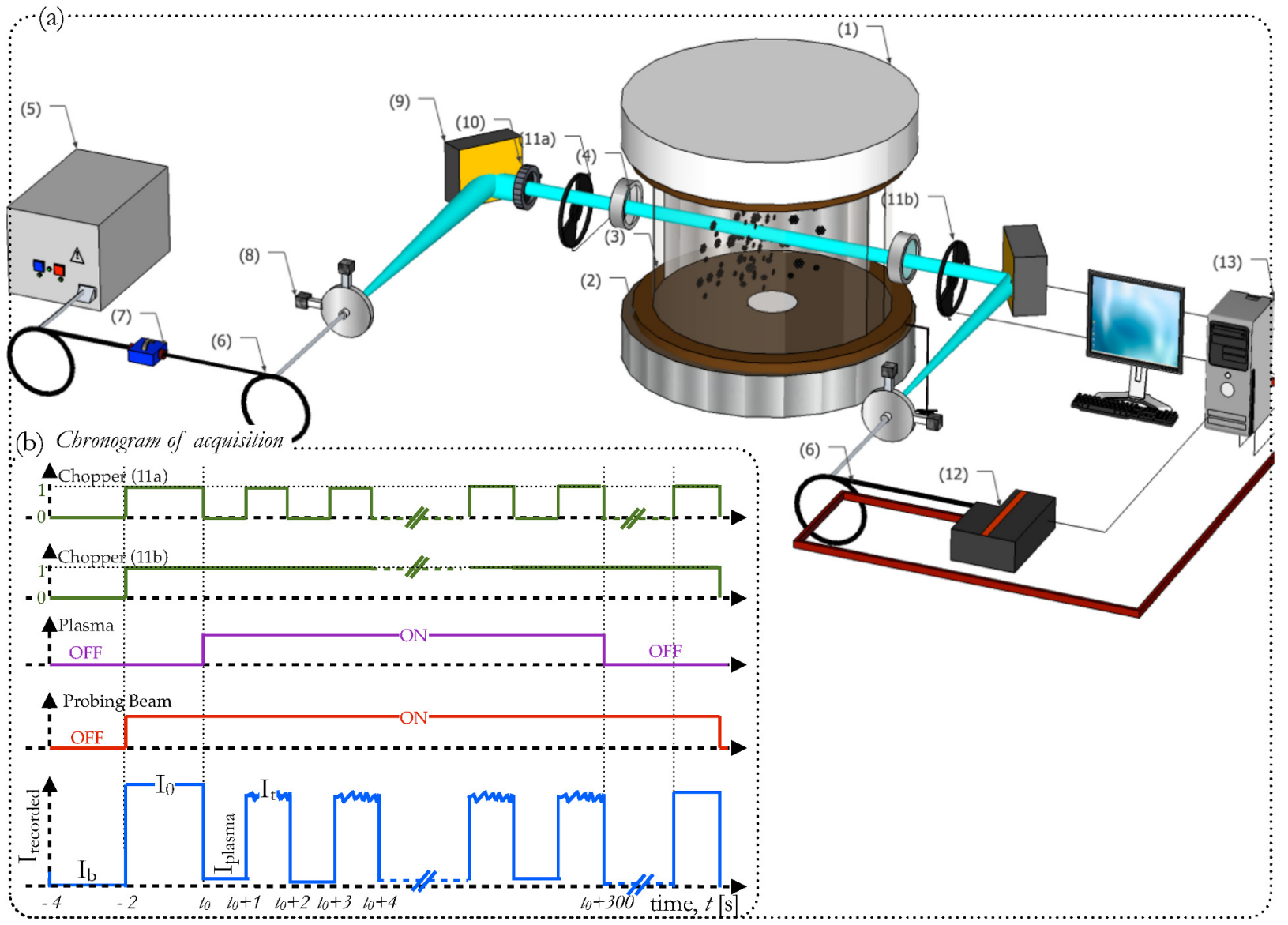

Figure A1. a) Schematic of the LES acquisition system: (1) tungsten cathode, (2) stainless-steal grounded anode, (3) glass half cylinders, (4) UV fused silica windows, (5) highly-stabilized Halogen-Deuterium lamp, (6) optical fibers, (7) on-line intensity attenuator, (8) achromatic coupling and collimating optics, (9) parabolic mirrors, (10) diaphragm, (11a - 11b) optical choppers, (12) UV-NIR spectrometer, (13) PC. b) Acquisition chronogram of the intensities

[16] Borowik L, Nuyen-Tran T, i Cabarrocas P R and Merlin T 2013 J. Appl. Phys. 114204305

[17] Fridman A A, Boufendi L, Hbid T, Potapkin B V and Bouchoule A 1996 J. Appl. Phys. 791303

[18] Howling A A, Sansonnens L, Dorier J L and Hollenstein C 1994 J. Appl. Phys. 75 1340-1353

[19] Bhandarkar U, Swihart M, Girshick S and Kortshagen U 2000 J. Phys. D: Appl. Phys. 332731

[20] Bouchoule A and Boufendi L 1993 Plasma Sources Sci. Technol. 2 204-213

[21] Hong S K and Winter J 2006 J. Appl. Phys. 100064303

[22] Kishor Kumar K., Couëdel L and Arnas C 2013 Phys. Plasmas 20043707

[23] Kishor Kumar K., Couëdel L and Arnas C 2014 Journal of Plasma Physics 80849

[24] Couëdel L, Kumar K K and Arnas C 2014 Phys. Plasmas 21123703

[25] Onofri F, Barbosa S, Touré O, Woniak M and Grisolia C 2013 J. Quant. Spectrosc. Radiat. Transfer 126160

[26] Gebauer G and Winter J 2003 New J. Phys. 538

[27] Greiner F, Carstensen J, Khler N, Pilch I, Ketelsen H, Knist S and Piel A 2012 Plasma Sources Sci. Technol. 21065005

[28] Baserga A, Russo V, Fonzo F D, Bailini A, Cattaneo D, Casari C, Bassi A L and Bottani C 2007 Thin Solid Films 5156465 - 6469 ISSN 0040-6090

[29] Onofri F and Barbosa S 2012 Chapter II: Optical particle characterization", in "Laser Metrology in Fluid Mechanics (London: Wiley-ISTE)

[30] Onofri F, Wozniak M and Barbosa S 2011 Contrib. Plasma Phys. 51 228-236

[31] Onofri F, Ren K and Grisolia C 2009 J. Nucl. Mat. 390-391 1093 - 1096 ISSN 0022-3115

[32] Palik E D 1985 Handbook of Optical Constants of Solid (Academic Press, London)

[33] Mikikian M, Cavarroc M, Couëdel L and Boufendi L 2006 Phys. Plasmas 13092103

[34] Gras-Marti A and Valles-Abarca J A 1983 J. Appl. Phys. 541071

[35] Mankelevich Y A, Olevanov M A and Rakhimova T V 2008 Plasma Sources Sci. Technol. 17015013

[36] Mankelevich Y, Olevanov M, Pal A, Rakhimova T, Ryabinkin A, Serov A and Filippov A 2009 Plasma Phys. Rep. 35(3) 191-199

[37] Annaratone B M, Elskens Y, Arnas C, Antonova T, Thomas H M and Morfill G E 2009 New J. Phys. 11103013

[38] Cavarroc M, Mikikian M, Tessier Y and Boufendi L 2008 Phys. Rev. Lett. 100045001

[39] Couëdel L, Mikikian M, Samarian A A and Boufendi L 2010 Phys. Plasmas 17083705 